\title{
AUTOMATIC CONTINUITY OF $n$-HOMOMORPHISMS BETWEEN TOPOLOGICAL ALGEBRAS
}

\author{
TAHER G. HONARY ${ }^{凶}$ and H. SHAYANPOUR
}

\author{
(Received 22 February 2010)
}

\begin{abstract}
A map $\theta: A \rightarrow B$ between algebras $A$ and $B$ is called $n$-multiplicative if $\theta\left(a_{1} a_{2} \cdots a_{n}\right)=\theta\left(a_{1}\right)$ $\theta\left(a_{2}\right) \cdots \theta\left(a_{n}\right)$ for all elements $a_{1}, a_{2}, \ldots, a_{n} \in A$. If $\theta$ is also linear then it is called an $n$ homomorphism. This notion is an extension of a homomorphism. We obtain some results on automatic continuity of $n$-homomorphisms between certain topological algebras, as well as Banach algebras. The main results are extensions of Johnson's theorem to surjective $n$-homomorphisms on topological algebras, a theorem due to C. E. Rickart in 1950 to dense range $n$-homomorphisms on topological algebras and two theorems due to E. Park and J. Trout in 2009 to $*$-preserving $n$-homomorphisms on $l m c *$-algebras.
\end{abstract}

2010 Mathematics subject classification: primary 46H40, 46H05; secondary 46K05.

Keywords and phrases: automatic continuity, $n$-homomorphism, topological algebras, $\operatorname{lmc}$ algebras, $Q$-algebras, $n$-involution, $*$-preserving $n$-homomorphisms.

\section{Introduction}

Let $A$ and $B$ be algebras and $n \geq 2$ be an integer. A mapping $\theta: A \rightarrow B$ is called $n$-multiplicative [anti- $n$-multiplicative] if

$$
\theta\left(a_{1} a_{2} \cdots a_{n}\right)=\theta\left(a_{1}\right) \theta\left(a_{2}\right) \cdots \theta\left(a_{n}\right)\left[=\theta\left(a_{n}\right) \theta\left(a_{2}\right) \cdots \theta\left(a_{1}\right)\right]
$$

for all elements $a_{1}, a_{2}, \ldots, a_{n} \in A$. If $\theta$ is also linear then it is called an $n$-homomorphism [anti- $n$-homomorphism]. Obviously, each homomorphism is an $n$-homomorphism for every $n \geq 2$, but the converse is not true, in general. For example, if $\varphi$ is a homomorphism then $\theta=-\varphi$ is a 3-homomorphism, which is not a homomorphism. For certain properties of 3-homomorphisms one may refer to [1]. If $A$ is unital with the unit element $e_{A}$ and $\theta: A \rightarrow B$ is an $n$-homomorphism then by [7, Proposition 2.2], there exists a homomorphism $\varphi: A \rightarrow B$ such that $\theta(a)=\theta\left(e_{A}\right) \varphi(a)$ for all $a \in A$. Furthermore, a 2-homomorphism is then just a homomorphism, in the usual sense. Thus we may assume in the following that $n \geq 3$. The concept of $n$-homomorphism was studied for complex algebras by Hejazian et al. in [7]. Fragoulopoulou [4, 5] in 1991 and 1993, and then Honary and Najafi [8] in 2008, obtained some results on the

(C) 2011 Australian Mathematical Publishing Association Inc. 0004-9727/2011 \$16.00 
automatic continuity of homomorphisms between topological $Q$-algebras. We extend some of these results to $n$-homomorphisms.

We now provide some notation and state some definitions and known results. For further details one can refer, for example, to $[2,6,9]$. If $A$ is a unital complex algebra with the unit $e_{A}$ then the spectrum of $a \in A$ is $\operatorname{sp}_{A}(a)=\left\{\lambda \in \mathbb{C}: \lambda e_{A}-a \notin \operatorname{Inv} A\right\}$, where Inv $A$ is the set of invertible elements of $A$. If $A$ is a nonunital complex algebra, then the spectrum of $a$ is

$$
\operatorname{sp}_{A}(a)=\{0\} \cup\left\{\lambda \in \mathbb{C} \backslash\{0\}: \frac{1}{\lambda} a \notin q-\operatorname{Inv} A\right\},
$$

where $q$-Inv $A$ is the set of quasi-invertible elements of $A$. If $A^{+}$is the unitization of $A$, then $\operatorname{sp}_{A}(a)=\operatorname{sp}_{A^{+}}((a, 0))$ and so $v_{A}(a)=v_{A^{+}}((a, 0))$ for all $a \in A$, where $v_{A}(a)$ is the spectral radius of $a$ with respect to the algebra $A$.

A left ideal $I$ of an algebra $A$ is a modular left ideal if there exists $u \in A$ such that $A\left(e_{A}-u\right) \subseteq I$, where $A\left(e_{A}-u\right)=\{x-x u: x \in A\}$. The Jacobson $\operatorname{radical} \operatorname{Rad}(A)$ of $A$ is the intersection of all maximal modular left ideals of $A$. The strong radical $\mathfrak{R}(A)$ of $A$ is the intersection of all maximal modular (two-sided) ideals of $A$. An algebra $A$ is called simple if $A^{2} \neq 0$ and if 0 and $A$ are the only ideals in $A$. An algebra $A$ is called semisimple whenever its $\operatorname{Jacobson} \operatorname{radical} \operatorname{Rad}(A)$ is trivial and it is called strongly semisimple if $\mathfrak{R}(A)$ is trivial.

A locally multiplicatively convex $(\operatorname{lm} c)$ algebra is a topological algebra whose topology is defined by a separating family $\mathcal{P}=\left(p_{\alpha}\right)$ of submultiplicative seminorms. A complete metrizable $l m c$ algebra is a Fréchet algebra. An $F$-algebra is a topological algebra whose underlying topological linear space is an $F$-space; in other words, the topology of an $F$-algebra is defined by a complete invariant metric. A Fréchet algebra is an $F$-algebra which is also an $l m c$ algebra. The topology of a Fréchet algebra $A$ can be generated by a sequence $\left(p_{n}\right)_{n \in \mathbb{N}}$ of separating submultiplicative seminorms, that is, $p_{n}(x y) \leq p_{n}(x) p_{n}(y)$ for all $n \in \mathbb{N}$ and $x, y \in A$, such that $p_{n}(x) \leq p_{n+1}(x)$ for all $x \in A$ and $n \in \mathbb{N}$.

An algebra $A$ equipped with an involution is called an involutive algebra, or a *-algebra. A topological *-algebra is a topological algebra with a continuous involution. If $\left(A,\left(p_{\alpha}\right)\right)$ is an involutive topological algebra with a family of seminorms $\left(p_{\alpha}\right)$ such that $p_{\alpha}\left(x^{*}\right)=p_{\alpha}(x)$ for all $x \in A$ and for every $\alpha$, then $A$ is clearly a topological $*$-algebra. If $A$ is an involutive algebra and $p$ is a seminorm on $A$, which satisfies the property $p\left(x^{*} x\right)=p(x)^{2}$ for all $x \in A$, then $p$ is called a $\mathrm{C}^{*}$-seminorm. The completion of an involutive topological algebra, whose topology is defined by a family of $\mathrm{C}^{*}$-seminorms, is called a locally $\mathrm{C}^{*}$-algebra. An $\operatorname{lmc}$ *-algebra is an involutive $l m c$ algebra with a family of seminorms $\mathcal{P}=\left(p_{\alpha}\right)$ such that $p_{\alpha}\left(a^{*}\right)=p_{\alpha}(a)$ for every $\alpha$ and all $a \in A$. If, moreover, $p_{\alpha}$ is a $\mathrm{C}^{*}$-seminorm for every $\alpha$, it is called an $\operatorname{lmc} \mathrm{C}^{*}$-algebra.

For a topological algebra $\left(A,\left(p_{\alpha}\right)\right)$, with a family of submultiplicative seminorms $\mathcal{P}=\left(p_{\alpha}\right)$, the completion of $A / \operatorname{ker} p_{\alpha}$ with respect to the norm $p_{\alpha}^{\prime}\left([x]_{\alpha}\right)=p_{\alpha}(x)$ is 
denoted by $A_{\alpha}$, where $[x]_{\alpha}=x+\operatorname{ker} p_{\alpha}$. Clearly, $A_{\alpha}$ is a Banach algebra. If $A$ is an lmc $\mathrm{C}^{*}$-algebra, then $A_{\alpha}$ is a (Banach) $\mathrm{C}^{*}$-algebra for every $\alpha$.

If $A$ and $B$ are involutive algebras, then an $n$-homomorphism $\theta: A \rightarrow B$ is called a *-preserving $n$-homomorphism if $\theta\left(x^{*}\right)=\theta(x)^{*}$ for all $x \in A$.

A topological algebra $A$ is advertibly complete if a Cauchy net $\left(a_{\alpha}\right)$ in $A$ converges in $A$ whenever, for some $b \in A$, both $a_{\alpha}+b-a_{\alpha} \cdot b$ and $a_{\alpha}+b-b \cdot a_{\alpha}$ converge to zero.

A topological algebra $A$ is a $Q$-algebra if the set of its quasi-invertible elements $(q-\operatorname{Inv} A)$ is open in $A$.

It is interesting to note that every topological $Q$-algebra is advertibly complete [ 9 , Theorem I.6.4]. Moreover, every complete topological algebra is also advertibly complete [9, p. 45].

PRoposition 1.1 [6, Theorem 4.6]. If $\left(B,\left(p_{\alpha}\right)\right)$ is an advertibly complete lmc algebra, then, for every $x \in B$,

$$
\begin{gathered}
\operatorname{sp}_{B}(x)=\bigcup_{\alpha} \operatorname{sp}_{B_{\alpha}}\left([x]_{\alpha}\right), \\
v_{B}(x)=\sup _{\alpha} v_{B_{\alpha}}\left([x]_{\alpha}\right)=\sup _{\alpha} \lim _{m \rightarrow \infty}\left(p_{\alpha}\left(x^{m}\right)\right)^{1 / m} .
\end{gathered}
$$

Let $A$ and $B$ be topological linear spaces, and let $\theta: A \rightarrow B$ be a linear mapping. The separating space of $\theta$ is defined by

$$
\mathfrak{S}(\theta)=\left\{b \in B: \exists \text { net }\left(a_{\delta}\right) \text { in } A \text { such that } a_{\delta} \rightarrow 0 \text { and } \theta\left(a_{\delta}\right) \rightarrow b\right\} .
$$

The separating space $\mathfrak{S}(\theta)$ is a closed linear subspace of $B$; moreover, if $A$ and $B$ are $F$-spaces, then, by the closed graph theorem, $\theta$ is continuous if and only if $\mathfrak{S}(\theta)=\{0\}$ [2, Proposition 5.1.2].

The following lemma has been proved by Ransford in [11], for unital Banach algebras, but it is also valid for nonunital algebras.

LEMMA 1.2. Let $B$ be an algebra, let $y \in B$, and suppose that $v_{B}\left(y^{\prime} y\right)=0$ for all $y^{\prime} \in B$. Then $y \in \operatorname{Rad}(B)$.

The following lemma, which will be used later, is also due to Ransford [11].

LEMMA 1.3. Let B be a Banach algebra, let $p(z)$ be a polynomial with coefficients in $B$, and let $R>0$. Then

$$
v_{B}(p(1))^{2} \leq \sup _{|z|=R} v_{B}(p(z)) \sup _{|z|=1 / R} v_{B}(p(z)) .
$$

\section{Extensions of Johnson's theorem for $\boldsymbol{n}$-homomorphisms on topological algebras}

We first state the following theorem, which appeared in [7, Proposition 2.2], and then deduce two useful results. 
THEOREM 2.1. Let $A$ be a unital algebra with the identity $e_{A}$, let $B$ be an algebra and $\theta: A \rightarrow B$ be an n-homomorphism. If $\psi: A \rightarrow B$ is defined by $\psi(x)=\theta\left(e_{A}\right)^{n-2} \theta(x)$ then $\psi$ is a homomorphism and $\theta(x)=\theta\left(e_{A}\right) \psi(x)$.

COROLlary 2.2. With the same hypotheses as in the theorem, if $\theta$ is surjective then $\psi$ is also surjective.

Proof. Clearly, $\theta\left(e_{A}\right)^{n-1} \theta(x)=\theta(x)$ for all $x \in A$. For every $y \in B$ there exists $x \in A$ such that $\theta(x)=y$. Moreover, there exists $t \in A$ such that $\theta(t)=\theta\left(e_{A}\right) \theta(x)$. Hence $\theta\left(e_{A}\right)^{n-2} \theta(t)=\theta\left(e_{A}\right)^{n-1} \theta(x)=\theta(x)$ and so $\psi(t)=\theta(x)=y$. Therefore, $\psi$ is surjective.

Corollary 2.3. Let $A$ and $B$ be topological algebras, where $A$ is unital. If $\theta: A \rightarrow$ $B$ is a dense range $n$-homomorphism, then $\psi$ is a dense range homomorphism.

PRoof. It is clear that $B$ is also unital and $e_{B}=\theta\left(e_{A}\right)^{n-1}$. Let $y \in B$. For $z=\theta\left(e_{A}\right) y$ there is a net $\left(x_{\alpha}\right)$ in $A$ such that $\theta\left(x_{\alpha}\right) \rightarrow z$. Hence

$$
\psi\left(x_{\alpha}\right)=\theta\left(e_{A}\right)^{n-2} \theta\left(x_{\alpha}\right) \rightarrow \theta\left(e_{A}\right)^{n-2} z=y .
$$

Since $y \in B$ is arbitrary, it follows that $\overline{\psi(A)}=B$.

Lemma 2.4. Let $A$ be an algebra, $\lambda \in \mathbb{C} \backslash\{0\}$ and $k \in \mathbb{N}$. If $a, d \in A$ and $\lambda \notin \operatorname{sp}_{A}\left(a^{k}\right)$ then there exists an element $c \in A$ such that $c\left(\lambda e_{A^{+}}-a^{k}\right)=d$.

PROOF. If $\lambda \notin \operatorname{sp}_{A}\left(a^{k}\right)$, then $c=d\left(\lambda e_{A^{+}}-a^{k}\right)^{-1} \in A^{+}$satisfies

$$
c\left(\lambda e_{A^{+}}-a^{k}\right)=d\left(\lambda e_{A^{+}}-a^{k}\right)^{-1}\left(\lambda e_{A^{+}}-a^{k}\right)=d .
$$

Since $c \in A^{+}$, there exist $\alpha \in \mathbb{C}$ and $b \in A$ such that $c=(b, \alpha)$ and so

$$
(d, 0)=(b, \alpha)\left[(0, \lambda)-\left(a^{k}, 0\right)\right]=(\lambda b, \lambda \alpha)-\left(b a^{k}+\alpha a^{k}, 0\right) .
$$

Thus $\lambda \alpha=0$ and hence $\alpha=0$, which shows that $c \in A$.

LEMMA 2.5. Let $\left(B,\left(p_{\alpha}\right)_{\alpha \in I}\right)$ be an lmc algebra, $\lambda \in \mathbb{C} \backslash\{0\}$ and $k \in \mathbb{N}$. If, for $b \in$ $B$, there exists an element $c \in B$ such that $c\left(\lambda e_{B^{+}}-b^{k}\right)=b$, then $\lambda \notin \operatorname{bd}\left(\operatorname{sp}_{B_{\alpha}^{+}}\left[b^{k}\right]_{\alpha}\right)$ for all $\alpha \in I$, where bd denotes the boundary (of a set) in the complex plane.

PRoOF. If $\lambda \in \operatorname{bd}\left(\operatorname{sp}_{B_{\alpha}^{+}}\left[b^{k}\right]_{\alpha}\right)$ for some $\alpha \in I$, then, by [2, Theorem 2.3.21(ii)], there exists a sequence $c_{n} \in \operatorname{Inv} B_{\alpha}^{+}$such that $\left\|c_{n}\right\|_{\alpha}=1$, where $\|\cdot\|_{\alpha}$ is the norm on $B_{\alpha}^{+}$, and

$$
\left(\left[\lambda e_{B^{+}}\right]_{\alpha}-\left[b^{k}\right]_{\alpha}\right) c_{n} \rightarrow 0 .
$$

Then by the hypothesis we have

$$
[c]_{\alpha}\left(\left[\lambda e_{B^{+}}\right]_{\alpha}-\left[b^{k}\right]_{\alpha}\right) c_{n}=[b]_{\alpha} c_{n} \rightarrow 0 .
$$

Since $\lambda \neq 0$ and $\lambda \in \operatorname{bd}\left(\operatorname{sp}_{B_{\alpha}^{+}}\left[b^{k}\right]_{\alpha}\right)$, it follows that $b \notin \operatorname{ker} p_{\alpha}$. Hence $\lim _{n \rightarrow \infty} c_{n}=0$, which is a contradiction. 
LEMMA 2.6. Let $A$ be an lmc algebra and $\left(B,\left(p_{\alpha}\right)_{\alpha \in I}\right)$ be an advertibly complete lmc algebra. If $\theta: A \rightarrow B$ is an n-homomorphism and $a \in A$, then

$$
\operatorname{bd}\left(\operatorname{sp}_{B_{\alpha}^{+}}\left(\left[\theta(a)^{n-1}\right]_{\alpha}\right)\right) \subseteq \operatorname{sp}_{A}\left(a^{n-1}\right) \cup\{0\},
$$

for all $\alpha \in I$. Moreover, $v_{B_{\alpha}}\left(\left[\theta(a)^{n-1}\right]_{\alpha}\right) \leq v_{A}\left(a^{n-1}\right)$ for all $\alpha \in I$ and hence

$$
v_{B}\left(\theta(a)^{n-1}\right) \leq v_{A}\left(a^{n-1}\right) .
$$

PROOF. Since $A$ is an $\operatorname{lm} c$ algebra and $\operatorname{sp}_{A^{+}}((a, 0))=\operatorname{sp}_{A}(a)$ for every $a \in A$, by [9, Corollary II.4.1], $\operatorname{sp}_{A}(a) \neq \varnothing$ for every $a \in A$. Suppose that $\lambda \neq 0$ such that $\lambda \notin \operatorname{sp}_{A}\left(a^{n-1}\right)$. By Lemma 2.4, for $d=a$, there exists an element $c \in A$ such that $a=c\left(\lambda e_{A^{+}}-a^{n-1}\right)=\lambda c-c a^{n-1}$. Hence

$$
\theta(a)=\theta\left(\lambda c-c a^{n-1}\right)=\theta(c)\left(\lambda e_{B^{+}}-\theta(a)^{n-1}\right) .
$$

From Lemma 2.5 , it follows that $\lambda \notin \operatorname{bd}\left(\operatorname{sp}_{B_{\alpha}^{+}}\left(\left[\theta(a)^{n-1}\right]_{\alpha}\right)\right)$ for all $\alpha \in I$ and hence that

$$
\operatorname{bd}\left(\operatorname{sp}_{B_{\alpha}^{+}}\left(\left[\theta(a)^{n-1}\right]_{\alpha}\right)\right) \subseteq \operatorname{sp}_{A}\left(a^{n-1}\right) \cup\{0\},
$$

for all $\alpha \in I$. It is now clear from Proposition 1.1 that $v_{B}\left(\theta(a)^{n-1}\right) \leq v_{A}\left(a^{n-1}\right)$.

It is interesting to note that the above lemma is also valid if $\theta$ is an anti-n-homomorphism.

THEOREM 2.7. Let $A$ be a unital topological $Q$-algebra and let $B$ be an advertibly complete semisimple lmc algebra. If $\theta: A \rightarrow B$ is a surjective n-homomorphism then $\theta$ has a closed graph.

Proof. By Corollary 2.2 we have $\psi(A)=B$, where $\psi(x)=\theta\left(e_{A}\right)^{n-2} \theta(x)$. By [8, Theorem 2.3], $\psi$ has a closed graph and hence $\theta(x)=\theta\left(e_{A}\right) \psi(x)$ also has a closed graph.

COROLlary 2.8. Let $A$ be a unital F-algebra which is also a $Q$-algebra and let $B$ be a semisimple Fréchet algebra. Then every surjective n-homomorphism $\theta: A \rightarrow B$ is automatically continuous.

An algebra $A$ is called factorizable if, for each $a \in A$, there exist $b, c \in A$ such that $a=b c$. If $A$ is not unital in the above theorem then we have the following result.

THEOREM 2.9. Let $A$ be an lmc $Q$-algebra and $B$ be a factorizable advertibly complete lmc semisimple algebra. Then every surjective $n$-homomorphism $\theta: A \rightarrow B$ has a closed graph.

PROOF. Let $\left(p_{\beta}\right)$ be a family of seminorms on $A$, and $\left(q_{\alpha}\right)$ be a family of seminorms on $B$. Denote by $B_{\alpha}$ the Banach algebra obtained by the completion of $B / \operatorname{ker} q_{\alpha}$ with respect to the norm $q_{\alpha}^{\prime}\left([b]_{\alpha}\right)=q_{\alpha}(b)$, for $b \in B$. It is enough to show that, for any net $\left(x_{\delta}\right)$ in $A$, if $x_{\delta} \rightarrow 0$ in $A$ and $\theta\left(x_{\delta}\right) \rightarrow y$ in $B$, then $y=0$. 
By the surjectivity of $\theta$, there exists $x \in A$ such that $\theta(x)=y$. We define a polynomial with coefficients in $B$ by $P_{\delta}(z)=z \theta\left(x_{\delta}\right)+\theta\left(x-x_{\delta}\right)$. Since $B_{\alpha}$ is a Banach algebra,

$$
v_{B_{\alpha}}\left(\left[P_{\delta}(z)\right]_{\alpha}\right) \leq q_{\alpha}\left(P_{\delta}(z)\right) \leq|z| q_{\alpha}\left(\theta\left(x_{\delta}\right)\right)+q_{\alpha}\left(\theta(x)-\theta\left(x_{\delta}\right)\right) .
$$

On the other hand, $[\theta]_{\alpha}=\theta+\operatorname{ker} q_{\alpha}$ is an $n$-homomorphism from $A$ to $B_{\alpha}$. By Lemma 2.6, for all $z \in \mathbb{C}$,

$$
v_{B_{\alpha}}\left(\left[P_{\delta}(z)\right]_{\alpha}^{n-1}\right) \leq v_{A}\left(\left(z x_{\delta}+\left(x-x_{\delta}\right)\right)^{n-1}\right) .
$$

Since $A$ is a $Q$-algebra, there exists $p_{\beta}$ with $v_{A} \leq p_{\beta}[6$, Theorem 6.18]. Hence

$$
v_{B_{\alpha}}\left(\left[P_{\delta}(z)\right]_{\alpha}^{n-1}\right) \leq p_{\beta}\left(\left(z x_{\delta}+\left(x-x_{\delta}\right)\right)^{n-1}\right) \leq\left(|z| p_{\beta}\left(x_{\delta}\right)+p_{\beta}\left(x-x_{\delta}\right)\right)^{n-1} .
$$

If $\lambda \in \operatorname{sp}_{B_{\alpha}}\left(\left[P_{\delta}(z)\right]_{\alpha}\right)$ then $\lambda^{n-1} \in \operatorname{sp}_{B_{\alpha}}\left(\left[P_{\delta}(z)\right]_{\alpha}^{n-1}\right)$ and so

$$
|\lambda| \leq\left(|z| p_{\beta}\left(x_{\delta}\right)+p_{\beta}\left(x-x_{\delta}\right)\right)
$$

Therefore,

$$
v_{B_{\alpha}}\left(\left[P_{\delta}(z)\right]_{\alpha}\right) \leq|z| p_{\beta}\left(x_{\delta}\right)+p_{\beta}\left(x-x_{\delta}\right) .
$$

Combining these estimates with Lemma 1.3, we deduce that, for all $\delta$ and all $R>0$,

$$
\begin{aligned}
v_{B_{\alpha}}\left([y]_{\alpha}\right)^{2} \leq & \sup _{|z|=1 / R}\left(|z| q_{\alpha}\left(\theta\left(x_{\delta}\right)\right)+q_{\alpha}\left(\theta(x)-\theta\left(x_{\delta}\right)\right)\right) \\
& \times \sup _{|z|=R}\left(|z| p_{\beta}\left(x_{\delta}\right)+p_{\beta}\left(x-x_{\delta}\right)\right)
\end{aligned}
$$

Since $x_{\delta} \rightarrow 0$ and $\theta\left(x_{\delta}\right) \rightarrow \theta(x)$, we obtain

$$
v_{B_{\alpha}}\left([y]_{\alpha}\right)^{2} \leq \frac{1}{R} q_{\alpha}(y) \cdot p_{\beta}(x) .
$$

By letting $R \rightarrow \infty$, it follows that $v_{B_{\alpha}}\left([y]_{\alpha}\right)=0$. Therefore, by Proposition 1.1, $v_{B}(y)=0$.

Now let $y^{\prime} \in B$. Since $B$ is a factorizable algebra there exist $y_{1}^{\prime}, \ldots, y_{n-1}^{\prime} \in B$ such that $y^{\prime}=y_{1}^{\prime} \cdots y_{n-1}^{\prime}$. Now we choose $x_{i}^{\prime} \in A, i=1, \ldots, n-1$, with $\theta\left(x_{i}^{\prime}\right)=y_{i}^{\prime}, i=$ $1, \ldots, n-1$. Then $x_{1}^{\prime} \cdots x_{n-1}^{\prime} x_{\delta} \rightarrow 0$ in $A$ and $\theta\left(x_{1}^{\prime} \cdots x_{n-1}^{\prime} x_{\delta}\right) \rightarrow y_{1}^{\prime} \cdots y_{n-1}^{\prime} y=$ $y^{\prime} y$ in $B$. Hence a repetition of the above argument shows that $v_{B}\left(y^{\prime} y\right)=0$. Since $y^{\prime}$ is arbitrary, by Lemma 1.2, it follows that $y \in \operatorname{Rad}(B)$ and hence $y=0$, as desired.

Corollary 2.10. Let $A$ and $B$ be Fréchet algebras such that $A$ is a $Q$-algebra and $B$ is factorizable and semisimple. Then every surjective $n$-homomorphism $\theta: A \rightarrow B$ is automatically continuous.

PROOF. This is immediate by the closed graph theorem. 
Since any unital algebra is factorizable, we also conclude the following result.

COROLlary 2.11. Let A be an lmc Q-algebra and B be a unital advertibly complete semisimple lmc algebra. Then every surjective $n$-homomorphism $\theta: A \rightarrow B$ has a closed graph.

Let $\left(A,\left(p_{\alpha}\right)\right)$ be an $\operatorname{lmc}$ algebra. A uniformly bounded left (right) approximate identity for $A$ is a net $\left\{e_{\gamma}\right\}_{\gamma \in \Lambda}$ such that:

(i) $\lim _{\gamma} e_{\gamma} a=a\left(\lim _{\gamma} a e_{\gamma}=a\right)$ for all $a \in A$;

(ii) $\sup _{\alpha} p_{\alpha}\left(e_{\gamma}\right)<\infty$ for all $\gamma \in \Lambda$.

REMARK 2.12. Many $l m c$ algebras which do not have an identity do have uniformly bounded left or right approximate identities. For example, every locally $\mathrm{C}^{*}$-algebra has a uniformly bounded approximate identity [6, Theorem 11.5]. Moreover, every Fréchet algebra, with a uniformly bounded left approximate identity, is factorizable [3, Theorem 4.1].

Hence we have the following result.

Corollary 2.13. Let $A$ be an lmc Q-algebra and $B$ be a semisimple Fréchet algebra with a uniformly bounded left approximate identity. Then every surjective $n$-homomorphism $\theta: A \rightarrow B$ has a closed graph. In particular, if $A$ and $B$ are Fréchet algebras such that $A$ is a $Q$-algebra and $B$ is a semisimple locally $C^{*}$-algebra, then every surjective $n$-homomorphism $\theta: A \rightarrow B$ is continuous.

PROPOSITION 2.14. Every anti-n-homomorphism on an lmc Q-algebra A onto an advertibly complete factorizable semisimple lmc algebra $B$ has a closed graph.

PROOF. Since $\operatorname{sp}_{B}\left(y^{\prime} y\right) \cup\{0\}=\operatorname{sp}_{B}\left(y y^{\prime}\right) \cup\{0\}$ for all $y, y^{\prime} \in B$ we have $v_{B}\left(y^{\prime} y\right)=$ $v_{B}\left(y y^{\prime}\right)$ for all $y, y^{\prime} \in B$. If we replace $y^{\prime} y$ by $y y^{\prime}$ in Lemma 1.2 then the lemma is still true. Since Lemma 2.6 is also valid for anti- $n$-homomorphisms, by the same argument as in the proof of Theorem 2.9 the result follows.

Let $A$ and $B$ be linear spaces over $K(\mathbb{R}$ or $\mathbb{C})$. A map $\theta: A \rightarrow B$ is conjugate-linear if

$$
\theta(\lambda x+y)=\bar{\lambda} \theta(x)+\theta(y), \quad x, y \in A, \lambda \in K .
$$

LEMMA 2.15. Let $A$ be an lmc algebra and $\left(B,\left(p_{\alpha}\right)_{\alpha \in I}\right)$ be an advertibly complete lmc algebra. If $\theta: A \rightarrow B$ is a conjugate-linear and n-multiplicative (or anti-nmultiplicative) mapping, then for every $a \in A$ and for each $\alpha \in I$,

$$
\left.\operatorname{bd}\left(\operatorname{sp}_{B_{\alpha}^{+}}\left(\left[\theta(a)^{n-1}\right]_{\alpha}\right)\right) \subseteq \overline{\operatorname{sp}_{A}\left(a^{n-1}\right)} \cup\{0\} \quad \text { (conjugate of } \operatorname{sp}_{A}\left(a^{n-1}\right)\right),
$$

where bd denotes the boundary (of a set) in the complex plane. Therefore,

$$
v_{B_{\alpha}}\left(\left[\theta(a)^{n-1}\right]_{\alpha}\right) \leq v_{A}\left(a^{n-1}\right),
$$

for all $\alpha \in I$ and hence $v_{B}\left(\theta(a)^{n-1}\right) \leq v_{A}\left(a^{n-1}\right)$.

PROOF. By modifying the proof of Lemma 2.6, the result follows. 
An $n$-involution on an algebra $A$ over $\mathbb{C}$ is a map $*: A \rightarrow A$ satisfying:

(i) $(a+b)^{*}=a^{*}+b^{*}$;

(ii) $\left(a_{1} a_{2} \cdots a_{n}\right)^{*}=a_{n}^{*} a_{n-1}^{*} \cdots a_{1}^{*}$;

(iii) $(\lambda a)^{*}=\bar{\lambda} a^{*}$;

(iv) $\underbrace{\left(\left(\left(a^{*}\right)^{*}\right)^{*} \cdots\right)^{*}}_{n}=a^{n *}=a$.

Note that every $n$-involution is conjugate-linear and anti- $n$-multiplicative. Hence we have the following result.

Proposition 2.16. Let A be a factorizable semisimple lmc $Q$-algebra. Then every $n$-involution on $A$ has a closed graph. If, in addition, $A$ is an F-algebra, then every $n$-involution on A is automatically continuous.

Proof. By Proposition 2.14, Lemma 2.15 and by modification of the proof of Theorem 2.9 the result follows.

\section{Extension of Rickart's theorem for dense range $\boldsymbol{n}$-homomorphisms on topological algebras}

We first extend [2, Proposition 5.1.3(i)] as follows.

Proposition 3.1. Let $A$ and $B$ be topological algebras and $\theta: A \rightarrow B$ be a dense range $n$-homomorphism such that $\theta(A)$ is factorizable. Then the separating space $\mathfrak{S}(\theta)$ is a closed (two-sided) ideal in $B$.

Proof. By [2, Proposition 5.1.2], the separating space $\mathfrak{S}(\theta)$ is a closed linear subspace of $B$. Let $b \in \mathfrak{S}(\theta)$ and $a \in A$. There exists a net $\left\{a_{\delta}\right\}$ in $A$ such that $a_{\delta} \rightarrow 0$ and $\theta\left(a_{\delta}\right) \rightarrow b$. Since $\theta(A)$ is a factorizable algebra, there are $a_{1}^{\prime}, \ldots, a_{n-1}^{\prime} \in A$ such that $\theta(a)=\theta\left(a_{1}^{\prime}\right) \cdots \theta\left(a_{n-1}^{\prime}\right)$. Since $a_{1}^{\prime} \cdots a_{n-1}^{\prime} a_{\delta} \rightarrow 0$ and $\theta\left(a_{1}^{\prime} \cdots a_{n-1}^{\prime} a_{\delta}\right) \rightarrow$ $\theta\left(a_{1}^{\prime}\right) \cdots \theta\left(a_{n-1}^{\prime}\right) b=\theta(a) b$, it follows that $\theta(a) b \in \mathfrak{S}(\theta)$. Similarly, $b \theta(a) \in \mathfrak{S}(\theta)$.

If $b^{\prime} \in B$ then there exists a net $\left\{a_{\beta}^{\prime}\right\}$ in $A$ such that $\theta\left(a_{\beta}^{\prime}\right) \rightarrow b^{\prime}$ and so $\theta\left(a_{\beta}^{\prime}\right) b \rightarrow$ $b^{\prime} b$. Since $\theta\left(a_{\beta}^{\prime}\right) b \in \mathfrak{S}(\theta)$ and $\mathfrak{S}(\theta)$ is closed, it follows that $b^{\prime} b \in \mathfrak{S}(\theta)$. Similarly, $b b^{\prime} \in \mathfrak{S}(\theta)$. Hence $\mathfrak{S}(\theta)$ is an ideal in $B$.

We now state a result due to Rickart in 1950, see [12, Theorem 6.18] and then extend it to more general cases.

Theorem 3.2. Let $A$ and $B$ be Banach algebras such that $A$ is unital and $B$ is strongly semisimple. Then every dense range homomorphism $\theta: A \rightarrow B$ is automatically continuous.

Proposition 3.3. Let $A$ and $B$ be Banach algebras such that $A$ is unital and $B$ is strongly semisimple. If $\theta: A \rightarrow B$ is a dense range $n$-homomorphism, then it is automatically continuous. 
Proof. By Corollary 2.3 we have $\overline{\psi(A)}=B$, where $\psi(x)=\theta^{n-2}\left(e_{A}\right) \theta(x), x \in A$, is a homomorphism. By Theorem 3.2, $\psi$ is continuous. Hence $\theta(x)=\theta\left(e_{A}\right) \psi(x)$ is also continuous.

In a unital algebra every ideal is modular. Moreover, in a unital $Q$-algebra every maximal ideal is closed. We now extend the above result to certain topological algebras.

THEOREM 3.4. Let $A$ and $B$ be lmc $Q$-algebras such that $B$ is a unital, strongly semisimple algebra. If $\theta: A \rightarrow B$ is a dense range $n$-homomorphism such that $\theta(A)$ is factorizable, then $\theta$ has a closed graph.

PROOF. It is enough to show that, for every net $x_{\delta} \in A$, if $x_{\delta} \rightarrow 0$ in $A$ and $\theta\left(x_{\delta}\right) \rightarrow y$ in $B$, then $y=0$. Let $M$ be a maximal ideal of $B$. Since $B$ is a unital $Q$-algebra, $M$ is closed and so, by $[6,6.14(3)], B / M$ is a $Q$-algebra. Since ideals in $B / M$ are in the form of $J / M$, where $J$ is an ideal in $B$ containing $M$, it is clear that the only ideals of $B / M$ are zero (that is, $M$ ) and $B / M$. Hence $B / M$ is simple. We now consider the $n$-homomorphism $\theta^{\prime}: A \rightarrow B / M$, which is the composition of $\theta$ and the natural map of $B$ onto $B / M$. By Proposition 3.1, $\mathfrak{S}\left(\theta^{\prime}\right)$ is an ideal of $B / M$. On the other hand, by Lemma 2.6 we have

$$
v_{B / M}\left(\theta^{\prime}(a)^{n-1}\right) \leq v_{A}\left(a^{n-1}\right) \quad(a \in A) .
$$

If $\lambda \in \operatorname{sp}_{B / M}\left(\theta^{\prime}(a)\right)$ then $\lambda^{n-1} \in \operatorname{sp}_{B / M}\left(\theta^{\prime}(a)^{n-1}\right)$ and so $v_{B / M}\left(\theta^{\prime}(a)\right) \leq v_{A}(a)$.

If $e_{B / M} \in \mathfrak{S}\left(\theta^{\prime}\right)$ then there exists a net $\left\{a_{\delta}\right\}$ in $A$ such that $a_{\delta} \rightarrow 0$ in $A$ and $\theta^{\prime}\left(a_{\delta}\right) \rightarrow e_{B / M}$ in $B$. Moreover,

$$
\begin{aligned}
1 & =v_{B / M}\left(e_{B / M}\right) \leq v_{B / M}\left(\theta^{\prime}\left(a_{\delta}\right)\right)+v_{B / M}\left(e_{B / M}-\theta^{\prime}\left(a_{\delta}\right)\right) \\
& \leq v_{A}\left(a_{\delta}\right)+v_{B / M}\left(e_{B / M}-\theta^{\prime}\left(a_{\delta}\right)\right) .
\end{aligned}
$$

Since $A$ and $B / M$ are $\operatorname{lmc} Q$-algebras, it follows from [9, Proposition III.6.2] that $v_{A}$ and $\nu_{B / M}$ are continuous at zero and so

$$
v_{A}\left(a_{\delta}\right)+v_{B / M}\left(e_{B / M}-\theta^{\prime}\left(a_{\delta}\right)\right) \rightarrow 0,
$$

which is a contradiction. Hence $e_{B / M} \notin \mathfrak{S}\left(\theta^{\prime}\right)$. Since $B / M$ is simple, it follows that $\mathfrak{S}\left(\theta^{\prime}\right)=M$, that is, $\theta^{\prime}$ is continuous and hence $\theta^{\prime}\left(x_{\delta}\right) \rightarrow 0$, which implies that $y \in M$. Since $M$ is an arbitrary maximal (modular) ideal, we conclude that $y \in \mathfrak{R}(B)$. Since $B$ is strongly semisimple, $y=0$. Therefore, $\theta$ has a closed graph.

COROLlary 3.5. Let $A$ and $B$ be Fréchet $Q$-algebras and let $B$ be unital and strongly semisimple. Suppose that $\theta: A \rightarrow B$ is a dense range $n$-homomorphism such that $\theta(A)$ is factorizable. Then $\theta$ is automatically continuous.

PROOF. By the closed graph theorem the result follows. 


\section{Automatic continuity of $\boldsymbol{n}$-homomorphism on $\operatorname{lmc} \mathrm{C}^{*}$-algebras}

For certain results on the automatic continuity of $n$-homomorphisms on Banach $\mathrm{C}^{*}$-algebras one may refer to [10], and for the automatic continuity of homomorphisms on $\operatorname{lmc} \mathrm{C}^{*}$-algebras one may refer to [4].

We now extend [10, Theorems 2.3 and 3.2], originally proved for Banach $\mathrm{C}^{*}$-algebras. For this purpose, we need the following useful lemmas.

LEMMA 4.1. Let $\left(B,\left(p_{\alpha}\right)_{\alpha \in I}\right)$ be an lmc $*$-algebra, $\lambda \in \mathbb{C} \backslash\{0\}$ and $k \in \mathbb{N}$. If, for $b \in B$, there exists an element $c \in B$ such that $c\left(\lambda e_{B^{+}}-\left(b^{*} b\right)^{k}\right)=b$, then $\lambda \notin$ $\operatorname{bd}\left(\operatorname{sp}_{B_{\alpha}^{+}}\left[\left(b^{*} b\right)^{k}\right]_{\alpha}\right)$ for all $\alpha \in I$.

PROOF. The proof is similar to the proof of Lemma 2.5.

LEMMA 4.2. Let $A$ be an lmc $*$-algebra and $\left(B,\left(p_{\alpha}\right)_{\alpha \in I}\right)$ be an advertibly complete lmc *-algebra. If $\theta: A \rightarrow B$ is a *-preserving $n$-homomorphism, $n=2 k+1$ and $a \in A$, then

$$
\operatorname{bd}\left(\operatorname{sp}_{B_{\alpha}^{+}}\left(\left[\left(\theta(a)^{*} \theta(a)\right)^{k}\right]_{\alpha}\right)\right) \subseteq \operatorname{sp}_{A}\left(\left(a^{*} a\right)^{k}\right) \cup\{0\},
$$

for all $\alpha \in I$. Moreover, $v_{B_{\alpha}}\left(\left[\left(\theta(a)^{*} \theta(a)\right)^{k}\right]_{\alpha}\right) \leq v_{A}\left(\left(a^{*} a\right)^{k}\right)$ for all $\alpha \in I$ and hence

$$
v_{B}\left(\left(\theta(a)^{*} \theta(a)\right)^{k}\right) \leq v_{A}\left(\left(a^{*} a\right)^{k}\right) .
$$

Proof. Let $\lambda \neq 0$ and $\lambda \notin \operatorname{sp}_{A}\left(\left(a^{*} a\right)^{k}\right)$. By Lemma 2.4 there exists $c \in A$ such that $c\left(\lambda e_{A^{+}}-\left(a^{*} a\right)^{k}\right)=a$. By applying Lemmas 2.6 and 4.1 the result follows.

TheOREM 4.3. Let $A$ be a topological $Q$-algebra, which is an lmc *-algebra with the family of seminorms $\mathcal{P}=\left(P_{\alpha}\right)$. Let $B$ be an $\operatorname{lmc} C^{*}$-algebra with the family of seminorms $\mathcal{Q}=\left(q_{\beta}\right)$. If $\theta: a \rightarrow B$ is a *-preserving $n$-homomorphism, then for each $q_{\beta}$ there exists a $p_{\alpha}$ such that $q_{\beta}(\theta(x)) \leq p_{\alpha}(x)$ for all $x \in A$. Hence $\theta$ is continuous on $A$.

Proof. Since $A$ is a $Q$-algebra, there is a $p_{\alpha}$ such that $v_{A} \leq p_{\alpha}$ [6, Theorem 6.18]. Since $B_{\beta}$ is a (Banach) $C^{*}$-algebra, by [2, Proposition 3.2.3],

$$
v_{B_{\beta}}\left([\theta(x)]_{\beta}^{*}[\theta(x)]_{\beta}\right)=q_{\beta}^{\prime}\left([\theta(x)]_{\beta}^{*}[\theta(x)]_{\beta}\right)=q_{\beta}\left(\theta(x)^{*} \theta(x)\right)=q_{\beta}(\theta(x))^{2} .
$$

Without loss of generality, we may assume that $B$ is complete. By Proposition 1.1, for every $\beta$ and for all $x \in A$, we have

$$
v_{B_{\beta}}\left(\left([\theta(x)]_{\beta}^{*}[\theta(x)]_{\beta}\right)\right) \leq v_{B}\left((\theta(x))^{*} \theta(x)\right) .
$$

We now consider two cases. First we assume that $n=2 k$. By Lemma 2.6, for every $x \in A$,

$$
\begin{aligned}
v_{B}\left(\theta\left(\left(x^{*} x\right)^{k}\right)^{n-1}\right) & \leq v_{A}\left(\left(x^{*} x\right)^{k(n-1)}\right) \leq p_{\alpha}\left(\left(x^{*} x\right)^{k(n-1)}\right) \\
& \leq\left(p_{\alpha}\left(x^{*} x\right)\right)^{k(n-1)} \leq p_{\alpha}(x)^{2 k(n-1)}
\end{aligned}
$$


Also

$$
\theta\left(\left(x^{*} x\right)^{k}\right)=\theta\left(x^{*} x \cdots x^{*} x\right)=\left(\theta\left(x^{*}\right) \theta(x)\right)^{k}=\left(\theta(x)^{*} \theta(x)\right)^{k} \quad \text { for all } x \in A .
$$

Hence $\lambda \in \operatorname{sp}_{B}\left((\theta(x))^{*} \theta(x)\right)$ implies that $\lambda^{k(n-1)} \in \operatorname{sp}_{B}\left(\theta\left(\left(x^{*} x\right)^{k}\right)^{n-1}\right)$. Consequently,

$$
v_{B}\left((\theta(x))^{*} \theta(x)\right) \leq p_{\alpha}(x)^{2} .
$$

From (4.1) and (4.2) we obtain $q_{\beta}(\theta(x)) \leq p_{\alpha}(x)$.

Next we assume that $n=2 k+1$. By Lemma 4.2 we have

$$
v_{B}\left(\left(\theta(x)^{*} \theta(x)\right)^{k}\right) \leq v_{A}\left(\left(x^{*} x\right)^{k}\right) \leq p_{\alpha}\left(\left(x^{*} x\right)^{k}\right) \leq p_{\alpha}(x)^{2 k} .
$$

If $\lambda \in \operatorname{sp}_{B}\left((\theta(x))^{*} \theta(x)\right)$ then $\lambda^{k} \in \operatorname{sp}_{B}\left((\theta(x))^{*} \theta(x)\right)^{k}$. Consequently,

$$
v_{B}\left((\theta(x))^{*} \theta(x)\right) \leq p_{\alpha}(x)^{2} .
$$

Now by (4.1) and (4.2) we have

$$
q_{\beta}(\theta(x))^{2} \leq v_{B}\left((\theta(x))^{*} \theta(x)\right) \leq p_{\alpha}(x)^{2} .
$$

Hence $q_{\beta}(\theta(x)) \leq p_{\alpha}(x)$ for all $x \in A$. It is now clear that this inequality implies the continuity of $\theta$ at zero and hence $\theta$ is continuous on $A$.

REMARK 4.4. It is clear that all results of this paper are valid for Banach algebras $A$ and $B$ if they have other properties required in each result.

\section{References}

[1] J. Bračič and S. Moslehian, 'On automatic continuity of 3-homomorphisms on Banach algebras', Bull. Malays. Math. Sci. Soc. (2) 30(2) (2007), 195-200.

[2] H. G. Dales, Banach Algebras and Automatic Continuity, London Mathematical Society Monograph, 24 (Clarendon Press, Oxford, 2000).

[3] P. G. Dixon, 'Automatic continuity of positive functionals on topological involution algebras', Bull. Aust. Math. Soc. 23 (1981), 265-281.

[4] M. Fragoulopoulou, 'Automatic continuity of $*$-morphisms between nonnormed topological $*-$ algebras', Pacific J. Math. 147(1) (1991).

[5] M. Fragoulopoulou, 'Uniqueness of topology for semisimple LFQ-algebras', Proc. Amer. Math. Soc. 117(4) (1993), 963-969.

[6] M. Fragoulopoulou, Topological Algebras with Involution (Elsevier, Amsterdam, 2005).

[7] M. Hejazian, M. Mirzavaziri and M. S. Moslehian, ' $n$-Homomorphism', Bull. Iranian Math. Soc. 31(1) (2005), 13-23.

[8] T. G. Honary and M. Najafi Tavani, 'Upper semicontinuity of the spectrum function and automatic continuity in topological $Q$-algebras', Note Mat. 28(2) (2008).

[9] A. Mallios, Topological Algebras, Selected Topics (North-Holland, Amsterdam, 1986).

[10] E. Park and J. Trout, 'On the nonexistence of nontrivial involutive $n$-homomorphisms of $\mathrm{C}^{*}$ algebras', Trans. Amer. Math. Soc. 361 (2009), 1949-1961. 
[11] T. J. Ransford, 'A short proof of Johnson's uniqueness-of-norm theorem', Bull. Lond. Math. Soc. 21 (1989), 487-488.

[12] A. M. Sinclair, Automatic Continuity of Linear Operators, London Mathematical Society Lecture Notes Series, 21 (Cambridge University Press, Cambridge, 1976).

TAHER G. HONARY, Faculty of Mathematical Sciences and Computer,

Tarbiat Moallem University, Tehran 1561836314, Iran

e-mail: honary@tmu.ac.ir

H. SHAYANPOUR, Faculty of Mathematical Sciences and Computer,

Tarbiat Moallem University, Tehran 1561836314, Iran

e-mail: h.shayanpour@yahoo.com 